\title{
CHALLENGES FOR SAMPLE PREPARATION AND ANALYSIS OF NEW MATRICES DURING THE OPCW PROFICIENCY TESTS
}

\author{
Nicoleta GRIGORIU*, Constantin Nicolae TOADER*, \\ Panaghia DELIU*, Dănuț MOȘTEANU** \\ *Scientific Research Center for CBRN Defense and Ecology, Bucharest, Romania, \\ **"Nicolae Bălcescu" Land Forces Academy, Sibiu, Romania \\ grig_nicole@yahoo.com, gabriel.epure@nbce.ro, cntoader@yahoo.com, \\ ghia_deliu@yahoo.com,dmosteanu@gmail.com
}

\begin{abstract}
In the OPCW Proficiency Tests usually two sets of three samples each (test sample, control, blank) are sent for analysis, with no indication for the type of the sample. Each set of samples can be prepared in a different matrix, with organic solvent, water and soil as the most frequent ones. Thus each sample has to be analyzed like a test sample. Participants are requested to identify any chemical contained in the schedules of the Chemical Weapons Convention plus all the degradation products that can originate from them; this involves millions of possible chemicals. Usually, a spiking level of $\approx 10 \mathrm{ppm}$ is applied. Only chemicals that are considered relevant within the scenario of the proficiency test are to be reported.

The choice of sample preparation method strongly depends on the composition of the sample and on the requirements for the different analytical techniques employed in the screening and analysis. Because the sample composition of proficiency test samples is unknown, no target compound sample preparation methods can be applied. New matrices, never used in a PT before, required participants to develop new or to adopt existing sample preparation methods. The new matrices from the $38^{\text {th }}$ OPCW PT were linked to food, biodiesel like used cooking oil methyl ester and a 2\% Agar-agar solution (solid gel). Aluminium metal and artificial rainwater chosen to provide matrix challenge for the $40^{\text {th }}$ OPCW PT. The paper provides the challenges that are generated by the new and complex matrices, how to work up gel sample, the tendency of biodiesel to form emulsions, how to sub-sample "metal fragments", and the method of extractions.
\end{abstract}

Keywords: OPCW, matrices, proficiency test, trichloronitromethane, 3-Quinuclidinyl benzilate

\section{Introduction}

In the OPCW [1] Proficiency Tests (PT) usually two sets of three samples each (test sample, control, blank) are sent for analysis, with no indication for the type of the sample. The test sample simulates a real sample collected during an inspection, contained an unknown number of spiking chemicals, each present at a concentration of $1 \mathrm{ppm}$ or greater. The control sample contained an unknown number of spiking chemicals, which mass spectral data is available in the OPCW Central Analytical Database, each present at a concentration of $5 \mathrm{ppm}$ or greater. The blank sample simulates the control sample prepared at the OPCW Laboratory and consists only of matrix similar to the real sample, with no chemicals relevant to the aim of the test. The goal of the tests is to report all 
chemicals relevant to the Chemical Weapons Convention [2] that are contained in the samples according to the tests scenario.

New matrices, never used in a PT before, were linked to food, biodiesel like used cooking oil methyl ester and a 2\% Agaragar solution (solid gel), and aluminium metal and artificial rainwater. The new matrices required to develop new or to adopt existing sample preparation methods.

\section{Experimental}

\subsection{Chemicals and materials}

The matrix of samples was an aqueous agar-agar gel, being the first time this material was used in an OPCW PT. The chemicals spiked in biodiesel (organic samples) made sample clean up a challenging work. The matrix of other samples was labelled as metal samples (aluminium metal), and it were samples as aqueous samples (artificial rainwater). As reagents in sample preparation procedures were used dichlorometane, methanol, acetonitrile, water of GC analytical grade, N,O-bis(trimethylsilyl) trifluoroacetamide (BSTFA), and $\mathrm{N}$-(tert-butyldimethylsilyl)N-methyltrifluoroacetamide (MTBSTFA).

\subsection{Sample preparation}

The gel sample was extracted with an organic solvent and water, centrifugal evaporation to dryness and silylation with BSTFA or MTBSTFA. It was performed also the methylation reaction and methanol extraction and silylation.

Chemicals spiked in biodiesel matrix made sample clean up a challenging work, because of the tendency of biodiesel to form emulsions. It was observed differences in hydrocarbon clean-up vs. clean-up of fatty acid methylesters in biodiesel. For the biodiesel matrix it were used as sample preparation procedures the clean up on silica-gel cartridge, silylation of the original sample, extraction with acetonitrile, extraction with water and silylation.

Another potential challenges was the determining how to sub-sample "metal fragments" and method of extraction. When the matrix is a solid (polymer, charcoal, soil, metal etc.), the spiking chemicals are absorbed in the substrate and liquid extractions need to be done in order to extract them from the matrix. In these cases, the spiking chemicals can be lost due to strong absorbance to the original matrix. Division of solid samples can be problematic and may cause loss of analytes of interest because of heterogeneity of the analytes in the sample material. The solid material sample is commonly extracted successively with an organic and an aqueous solvent. Our laboratory chooses to work with the whole metal fragment, not divide it. We start with the extraction with dichloromethane for analysis of nonpolar CWC-related chemicals. The organic extract was also silylated, because it might be possible that the extract contains polar CWC-related chemicals soluble in the used solvent. The aqueous extract is analysed for polar CWC-related chemicals such as alkylphosphonic and alkyl thiophosphonic acids, thiodiglycol, and amino alcohols. [3] The aqueous samples (with matrix as artificial rainwater) were extracted with organic solvent, concentration and silylation with BSTFA or MTBSTFA. [4]

\subsection{Instrumentation and detection of spiking chemicals}

GC/MS separations were performed on a Thermo Electron Corporation chromatograph GC Focus equipped with a mass detector DSQII. The GC was fitted with a $30 \mathrm{~m} \times 0.25 \mathrm{~mm}$ I.D. TR-5MS bonded phase column, $0.25 \mu \mathrm{m}$ film thickness (Thermo Fisher Scientific, U.S.A). Helium was used as the carrier gas at a column head pressure of $12 \mathrm{psi}$. The oven temperature was held initially at $40^{\circ} \mathrm{C}$ for $2 \mathrm{~min}$, programmed from $40^{\circ} \mathrm{C}$ to $300^{\circ} \mathrm{C}$ at $10^{\circ} \mathrm{C} \mathrm{m^{-1 }}$, and held at $280^{\circ} \mathrm{C}$ for 10 min. Splitless injections of $1 \mu \mathrm{L}$ volume were made using a Thermo Electron Corporation AI800 autosampler.

The electron ionization MS operating conditions were as follows: ion source 
pressure approximately $1.5 \times 10^{-5}$ torr; source temperature, $250^{\circ} \mathrm{C}$; electron energy, $70 \mathrm{eV}$; and electron multiplier voltage +400 $\mathrm{V}$ relative to the autotune setting.

\section{Results and discussions}

With no treatment of the biodiesel matrix it was reported trichloronitromethane $\left(\mathrm{Cl}_{3} \mathrm{CNO}_{2}\right.$, chloropicrine, Schedule 3.A.04., CAS no. 76-06-2) and dimethyl methylphosphonate $\left(\mathrm{C}_{3} \mathrm{H}_{9} \mathrm{O}_{3} \mathrm{P}\right.$, Schedule 2.B.04., CAS no. 756-79-6) in the sample that supported the purification on a silica cartridge. EI mass spectra of the trichloronitromethane confirming by the reference chemical can be shown in the Figure 1. EI chromatogram supporting identification of 3-Quinuclidinyl benzilate (BZ), from a metal fragment is presented in the Figure 2 and the mass spectrum of the 3-Quinuclidinyl benzilate confirming by the reference chemical from the laboratory synthesis can be shown in the Figure 3.

In this organic extract, the laboratory reported also the methyl benzilate, which is a non-scheduled reportable chemical as a precursor of the schedule chemical 3Quinuclidinyl benzilate. The silylated organic extract showed 2,2-Diphenyl-2hydroxyacetic acid (Benzilic acid). The chemicals that were found in a metal sample are presented in the Figure 4. Methyl benzilate and benzilic acid were categorised as non-scoring chemicals.

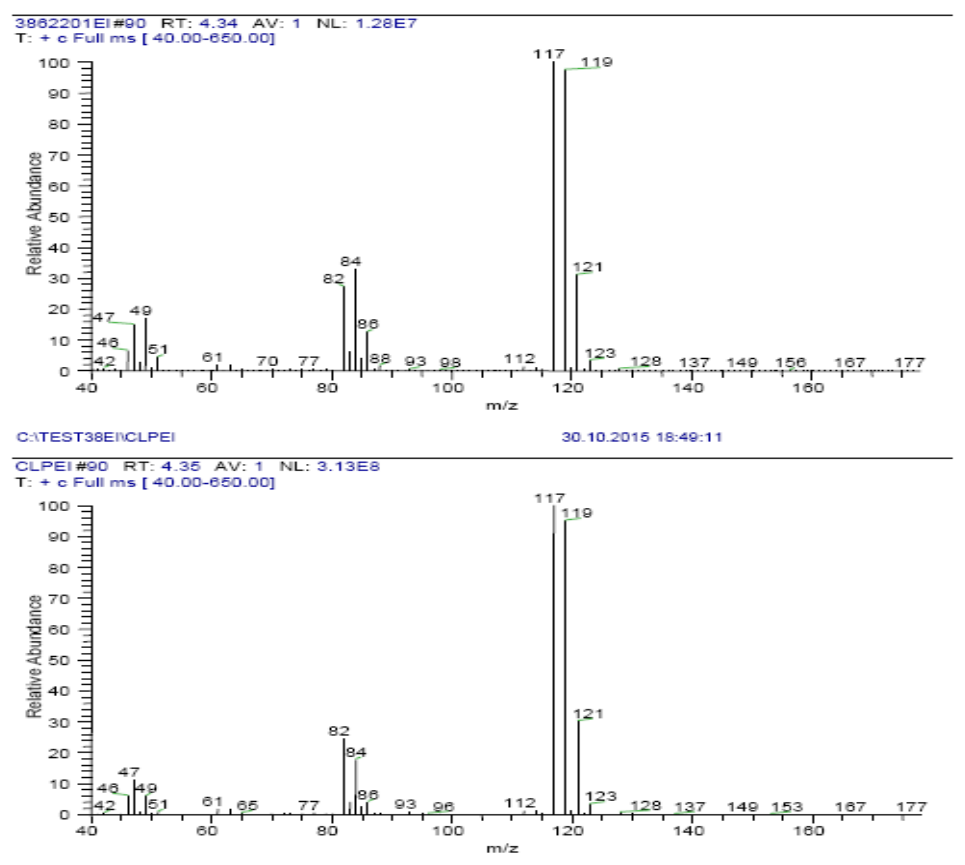

Figure 1: EI mass spectra of trichloronitromethane (top) and reference chemical from the laboratory synthesis (bottom) 


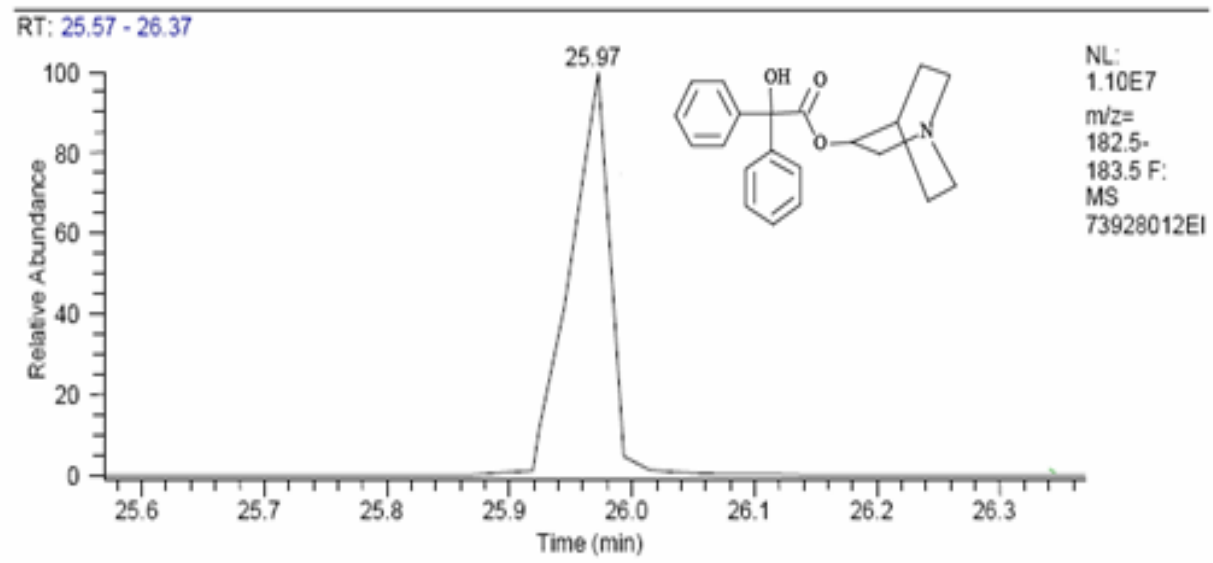

Figure 2: EI extracted ( $\mathrm{m} / \mathrm{z}$ 183) chromatogram supporting identification of 3-Quinuclidinyl benzilate, from a metal fragment, retention time of 25.97 minutes

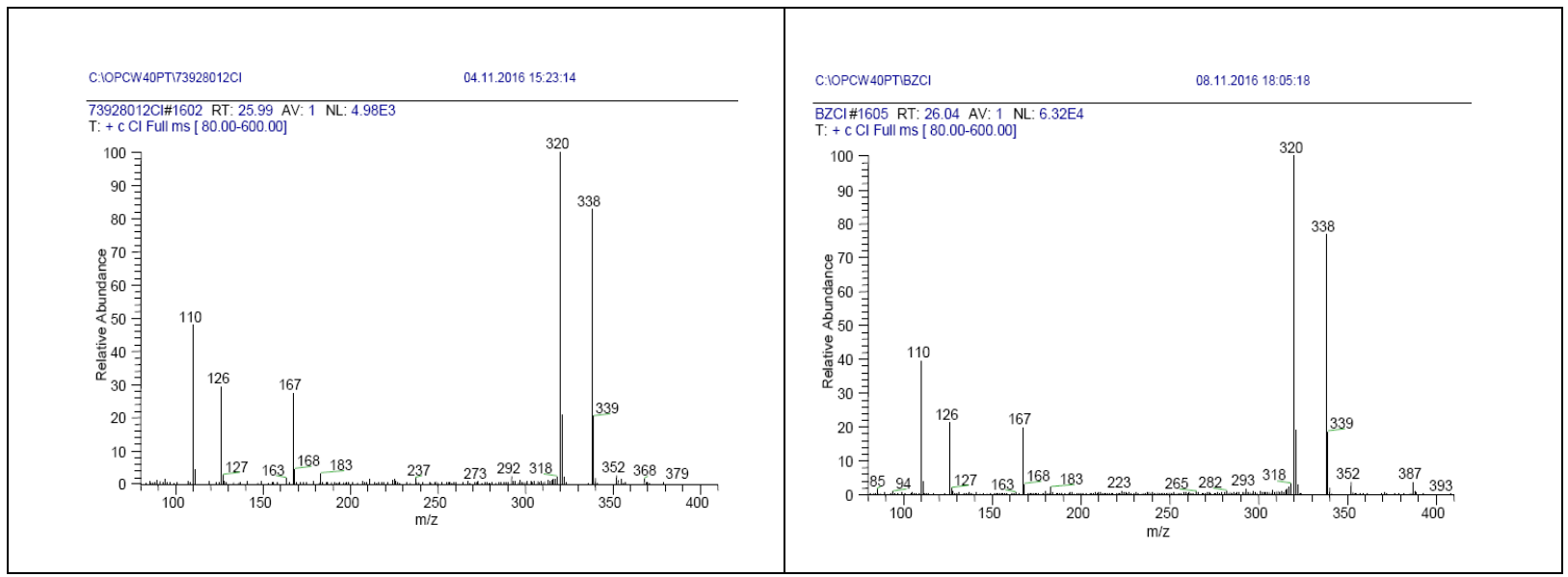

Figure 3: EI mass spectra of 3-Quinuclidinyl benzilate (MW 337) (left) and reference chemical (right)

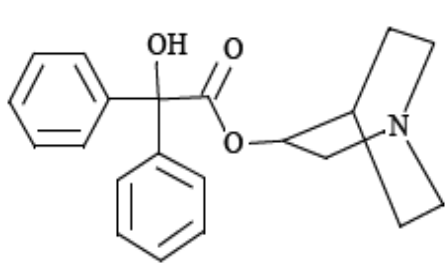

$\mathrm{BZ}$

[6581-06-2]

$\mathrm{C}_{21} \mathrm{H}_{24} \mathrm{O}_{3} \mathrm{~N}$

MW 338

*Schedule 1.A.03

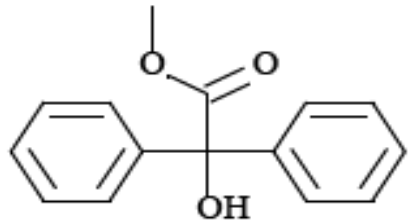

Methyl benzilate

[76-89-1]

$\mathrm{C}_{15} \mathrm{H}_{14} \mathrm{O}_{3}$

MW 242

Non-Scheduled

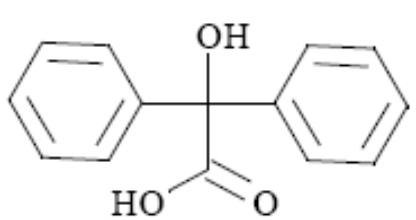

Benzilic acid

[76-93-7]

$\mathrm{C}_{14} \mathrm{H}_{12} \mathrm{O}_{3}$

MW 228

Schedule 2.B.08

*Schedule codes are from the Chemical Weapons Convention (CWC)

Figure 4: Spiking chemicals reported in a metal fragment sample. Methyl benzilate and benzilic acid were categorised as non-scoring chemicals 


\section{Conclusions}

The choice of sample preparation method strongly depends on the composition of the sample and on the requirements for the different analytical techniques employed in the screening and analysis. Because the sample composition of proficiency test samples is unknown, no target compound sample preparation methods can be applied.
New and complex matrices, such as biodiesel or solid gel, aluminium metal or artificial water were selected to provide matrix challenge for how to work up the samples. Silylation and methylation of the spiking chemicals can form derivatives as well as using aqueous solvents cause the chemicals to hydrolyse to chemicals that are not in the spiking chemicals list of the proficiency tests.

\section{References}

[1] The Organisation for the Prohibition of Chemical Weapons, http://www.opcw.org/.

[2] Convention on the Prohibition of the Development, Production, Stockpiling, and Use of Chemicals Weapons and their Destruction, signed in Paris in January 1993. Printed and distributed by the Provisional Technical Secretariat for the Preparatory Commission for the Organisation for the Prohibition of Chemicals Weapons. Depositary of the Convention is the Secretary -General of the United Nations.

[3] P. Vanninen. Recommended operating procedures for analysis in the verification of chemical disarmament. The Ministry for Foreign Affairs of Finland. University of Helsinki, 2011.

[4] Koskela, H., Grigoriu, N., Vanninen, P., Screening and identification of organophosphorus compounds related to the Chemical Weapons Convention with 1D and 2D NMR Spectroscopy. American Chemical Society, Analytical Chemistry, vol. 78 (11), $3715-3722,2006$. 